\title{
Modeling solvent dynamics in polymers with solvent-filled cavities
}

\author{
Michele Curatolo $^{1}$. Paola Nardinocchi ${ }^{1}$ (D) . Luciano Teresi ${ }^{2}$
}

Received: 6 August 2020 / Accepted: 26 August 2020 / Published online: 6 October 2020

(C) The Author(s) 2020

\begin{abstract}
Dynamics of solvent release from polymer gels with small solvent-filled cavities is investigated starting from a thermodynamically consistent and enriched multiphysics stress-diffusion model. Indeed, the modeling also accounts for a new global volumetric constraint which makes the volume of the solvent in the cavity and the cavity volume equal at all times. This induces a characteristic suction effect into the model through a negative pressure acting on the cavity walls. The problem is solved for gel-based spherical microcapsules and microtubules. The implementation of the mathematical model into a finite element code allows to quantitatively describe and compare the dynamics of solvent release from full spheres, hollow spheres, and tubules in terms of a few key quantities such as stress states and amount of released solvent under the same external conditions.
\end{abstract}

Keywords Polymer gels $\cdot$ Microcapslules dynamics $\cdot$ Solvent release $\cdot$ Suction pressure

\section{Introduction}

In the last years, solvent release from polymer gels has been intensively studied, as it is able to drive quite large deformations in polymer-based structures. Solvent release in response to specific stimuli is used in multi-responsive materials to meet clearly defined functional demands such as the onset of specific deformation patterns and the delivery of fixed amounts of solvent to the external environment. Multifunctional devices based on these multi-responsive materials are common in both nature and industries [1-8]. Solvent release drives a wide variety of deformations in multi-responsive bulk materials, depending on material architecture, boundary conditions, and external stimuli, only to cite a few key factors [4, 9-14]. On the other hand, the dynamics of the release process depends on the deformations which can significantly affect the rate of release; then, its control is as important as the control of the shape changes induced by the release in polymer-based structures.

Solvent release processes have been largely studied within the frame of the so-called stress-diffusion models which view the solvent-polymer mixture as a single homogenized continuum body allowing for a mass flux of the solvent [13, 15-18]. Typically, stress-diffusion models are based on the Flory-Rehner constitutive theory which describes the thermodynamics of the solvent-polymer mixture. Mostly, they deal with the analysis of the steady response of polymer gels under constraint and applied forces [11, 19-24]; however, the transient dynamics occurring during swelling or drying processes have been studied, too [13, 15, 17, 18, 25-27].

A different story has been going on when small solvent-filled cavities are present in the bulk polymer: solvent release comes from bulk as well as from cavities and the release changes the size of cavities which, on its turn, depends on the amount of released solvent. The process has been recently observed in the solvent-filled micro-cavities which are the elementary

Michele Curatolo

michele.curatolo@uniroma1.it

Paola Nardinocchi

paola.nardinocchi@uniroma1.it

Luciano Teresi

teresi@uniroma3.it

1 Sapienza Università di Roma, Rome, Italy

2 Università Roma Tre, Rome, Italy 
units of fern sporangium $[3,5]$. Therein, due to dehydration, the solvent is released from both the walls of the elementary units and the cavities and a shooting mechanism allowing for seed dispersal is produced when solvent cavitation is attained within the cavities.

In [28], the release process from a filled cavity has been studied in a partially constrained gel subject to traction, within the context of stress-diffusion theories. As usual, the driving force of the process is the change of the chemical potential of the environment which determines a change of chemical potential of the solvent in the gel. The proposed quasi-static analysis is controlled by the above change; the volume of the cavity, which is filled with an incompressible fluid whose volume is controlled by changing the temperature, is a further control parameter of the process. The problem is solved in two steps: first, the deformation of the gel caused by the change of the chemical potential of the solvent and by loads is evaluated at fixed cavity volume; then, the cavity volume is changed by changing the thermal expansion of the fluid filled in it. The variation of the size of a small cavity inside a swollen elastomer when environmental humidity changes and drying processes take place has been studied also in [29, 30] under different constraints and loading conditions. Therein, the steady analyses show that, starting from an initial swelling state, the cavity shrinks with the increase of humidity while the cavity grows with the decrease of humidity and the deformation state in the swollen elastomer for different humidity is evaluated. Differently from the model proposed in [28], the cavity volume freely changes at the different equilibrium states corresponding to different values of the environmental humidity.

A first attempt to describe the dynamics of the solvent release from a filled cavity other than from the bulk has been established in [31], where a study inspired by the observations in $[3,5]$ has been presented. It has been shown as dynamics of solvent release from cavities filled with incompressible solvents, at any time before the onset of cavitation, is strongly driven by the condition that the volume of the cavity has to match the volume of the water it contains. In [31], this condition has been interpreted as a global constraint which is enforced by adding a term to the total potential energy. Correspondingly, the Lagrange multiplier enforcing the constraint identifies the inner pressure which the solvent inside the cavity and the cavity walls exchange one with another. The evolving inner pressure is a key element of the model which makes it truly different from standard stress-diffusion models in the absence of filled cavities. However, in [31], it was assumed that the chemical potential of the solvent which fills the cavity cannot change during the dynamics, neglecting the change in the chemical potential due to the inner pressure. As a consequence, the system cannot attain any steady states but goes on de-hydrating; the evolution of the system is halted when the inner pressure gets the typical values of water cavitation.

In the present paper, we wish to make some progress towards addressing this question; we deal with the dynamics of water fluxes from hydrogel cavities induced by a change in the environmental conditions. As the well-known incompressibility constraint requires that any change in volume of a gel must be accompanied by uptake or release of solvent and holds everywhere and at all times, the so-called suction effect requires that the volume of the solvent in the cavity and the cavity volume must be the same at all time [31]. In our model, the reaction to the volumetric constraint is the inner pressure exerted by the solvent which fills the cavity on the cavity walls. The inner pressure, under some circumstances which will be discussed in the paper, may also attain negative values $[3,5,14]$; in this case, it determines a change in the chemical potential of the solvent which may attain the values of the chemical potential of the environment, so determining a steady state of the system. The above circumstances are identified by material parameters and have driven the distinction between poorly and highly swollen gels. The first ones mainly release solvent from the cavity and pressure in the cavity walls takes negative values; by contrast, highly swollen hydrogels mainly release solvent from the walls and pressure does not take negative values.

The control of solvent release from the capsule is achieved through an accurate change of the outer chemical potential which is assumed to depend on the chemical conditions of the outer environment. We show as, at the same changes of the outer chemical potential, the amount of released solvent is smaller and smaller and can be controlled by the differential changes of the outer chemical potential, a condition which is relevant in drug release applications. We also show the difference in solvent release from a full sphere and a capsule during the dynamics of the process; we also show the difference between the two situations in terms of stress state. Finally, a comparison between solvent dynamics in spherical microcapsules and cylindrical microtubules is also presented in the Appendices A and B.

\section{Stresses and diffusion in polymer gels}

Swelling and shrinking of polymer gels can be described through a nonlinear field theory which views the solvent-polymer mixture as a homogenized continuum body, allowing for a mass flux of the solvent $[15,17-19,25]$. In the following, we shortly review the key elements of the model originally presented in Ref. [18] and then improved in Refs. [14, 27, 31, 32] with special emphasis on swelling and shrinking dynamics. 
Usually, the reference state of a polymer body is identified by its dry state $\mathcal{B}_{d}$; we denote with $X_{d} \in \mathcal{B}_{d}$ a material point and with $t \in \mathcal{T}$ an instant of the time interval $\mathcal{T}$. The displacement field $\mathbf{u}_{d}\left(X_{d}, t\right)\left(\left[\mathbf{u}_{d}\right]=\mathrm{m}\right)$ from $\mathcal{B}_{d}$ gives the actual position $x$ at time $t$ of the point $X_{d}: x=f\left(X_{d}, t\right)=X_{d}+\mathbf{u}_{d}\left(X_{d}, t\right)$ and the molar solvent concentration per unit dry volume $c_{d}\left(X_{d}, t\right)\left(\left[c_{d}\right]=\mathrm{mol} / \mathrm{m}^{3}\right)$ gives the number of moles per unit dry volume. Displacement $\mathbf{u}_{d}$ and concentration $c_{d}$ fields are the state variables of our multiphysics problem and are coupled by the volumetric constraint ${ }^{1}$

$J_{d}=\operatorname{det} \mathbf{F}_{d}=\hat{J}_{d}\left(c_{d}\right)=1+\Omega c_{d} \quad$ with $\quad \mathbf{F}_{d}=\nabla f=\mathbf{I}+\nabla \mathbf{u}_{d}$,

which implies that any change in volume of the gel is accompanied by uptake or release of solvent where $\Omega$ denotes the molar volume of the solvent $\left([\Omega]=\mathrm{m}^{3} / \mathrm{mol}\right)$.

The thermodynamics of the model is based on the Flory-Rehner free energy representation $[33,34]$ which assumes that the free energy $\psi$ per unit dry volume is additively decomposed into an elastic component $\psi_{e}$ which depends on $\mathbf{F}_{d}$ and a polymer-solvent mixing energy $\psi_{m}$ which depends on $c_{d}$. The corresponding relaxed free energy $\psi_{r}$ includes the volumetric constraint which involves the pressure $p$ representing the reaction to the volumetric constraint. The latter maintains the volume change $J_{d}$ due to the displacement equal to the one due to solvent content $\hat{J}\left(c_{d}\right)$. The constitutive equations for the reference (also called Piola-Kirchhoff) stress $\mathbf{S}_{d}\left(\left[\mathbf{S}_{d}\right]=\mathrm{N} / \mathrm{m}^{2}\right)$ and the chemical potential $\mu$ of the solvent within the polymer $([\mu]=\mathrm{J} / \mathrm{mol})$ come from standard thermodynamic arguments [35] and prescribe that

$\mathbf{S}_{d}=\hat{\mathbf{S}}_{d}\left(\mathbf{F}_{d}\right)-p \mathbf{F}_{d}^{*} \quad$ with $\quad \hat{\mathbf{S}}_{d}\left(\mathbf{F}_{d}\right)=\frac{\partial \psi_{e}}{\partial \mathbf{F}_{d}}$,

and

$\mu=\hat{\mu}\left(c_{d}\right)+p \Omega \quad$ with $\quad \hat{\mu}\left(c_{d}\right)=\frac{\partial \psi_{m}}{\partial c_{d}}$.

The term $\mathbf{F}_{d}^{\star}=J_{d} \mathbf{F}_{d}^{-T}$ denotes the adjugate of the deformation gradient. From Eq. 2.2, the (Cauchy) stress $\mathbf{T}$ can be evaluated; it holds: $\left(1 / J_{d}\right) \mathbf{T}=\mathbf{S}_{d} \mathbf{F}^{T}$. In Eq. 2.3 , the constitutively determined component $\hat{\mu}\left(c_{d}\right)$ of the chemical potential can be interpreted as the osmotic pressure, whereas the term $p \Omega$ is the mechanical contribution to the chemical potential [13]. Finally, it is worth noting that the pressure term in both the constitutive equations for the stress and the chemical potential makes the elastic and diffusive problem strongly coupled also from a dynamical point of view.

The Flory-Rehner thermodynamic model, largely used in literature when continuum models of gels are considered [15$18,20]$, assumes a neo-Hookean free elastic energy and a mixing free energy derived by statistical mechanics procedures. The free energies (see for details $[14,17,18,32])$ include the physical parameters $G\left([G]=\mathrm{J} / \mathrm{m}^{3}\right.$ ) being the shear modulus of the dry polymer, $\mathcal{R}([\mathcal{R}]=\mathrm{J} /(\mathrm{K} \mathrm{mol}))$ the universal gas constant, $T([T]=\mathrm{K})$ the temperature, and $\chi$ the nondimensional Flory parameter. Equations 2.2-2.3 together with the assumed free energies deliver the constitutive equations for the dry-reference stress $\hat{\mathbf{S}}_{d}\left(\mathbf{F}_{d}\right)$ and for chemical potential $\hat{\mu}\left(c_{d}\right)$ in the form

$\hat{\mathbf{S}}_{d}\left(\mathbf{F}_{d}\right)=G \mathbf{F}_{d} \quad$ and $\quad \hat{\mu}\left(c_{d}\right)=\hat{\mu}\left(J_{d}\right)=\mathcal{R} T\left(\log \frac{J_{d}-1}{J_{d}}+\frac{1}{J_{d}}+\frac{\chi}{J_{d}^{2}}\right)$,

where the volumetric constraint (2.1) has been exploited to write the chemical potential in terms of $J_{d}$. A representation of the reference solvent flux $\mathbf{h}_{d}\left(\left[\mathbf{h}_{d}\right]=\mathrm{mol} /\left(\mathrm{m}^{2} \mathrm{~s}\right)\right)$ which is consistent with the dissipation principle is

$\mathbf{h}_{d}=\mathbf{h}_{d}\left(\mathbf{F}_{d}, c_{d}, p\right)=-\mathbf{D}_{d}\left(\mathbf{F}_{d}, c_{d}\right) \nabla\left(\hat{\mu}\left(c_{d}\right)+p \Omega\right)$,

provided that the diffusion tensor $\mathbf{D}_{d}=\mathbf{D}_{d}\left(\mathbf{F}_{d}, c_{d}\right)\left(\left[\mathbf{D}_{d}\right]=\mathrm{mol}^{2} /(\mathrm{s} \mathrm{m} \mathrm{J})\right)$ be positive definite. ${ }^{2}$ We assume that diffusion remains always isotropic during any process and increases with solvent concentration. It drives a representation formula of $\mathbf{D}_{d}$ as

$\mathbf{D}_{d}\left(\mathbf{F}_{d}, c_{d}\right)=\frac{D}{\mathcal{R} T} c_{d} \mathbf{C}_{d}^{-1}, \quad \mathbf{C}_{d}=\mathbf{F}_{d}^{T} \mathbf{F}_{d}$,

with $D\left([D]=\mathrm{m}^{2} / \mathrm{s}\right)$ the diffusivity $[15,17,25]$.

\footnotetext{
${ }^{1}$ It is often called incompressibility constraint as it is due to the incompressibility of both solvent and polymer.

${ }^{2}$ The same physics can be characterized in terms of actual quantities by writing $\mathbf{h}_{s}=-\mathbf{D}_{s}\left(\mathbf{F}_{d}, c_{s}\right) \operatorname{grad} \mu_{s}$. In this latter formula, since the chemical potential is not a density, the spatial to material transformation involves just the change of variable and $\mu=\mu_{s} \circ f$; on the contrary, the spatial concentration $c$ denotes the number of solvent moles, reckoned per unit current and $c_{d}=J(c \circ f)$. Finally, the differential operator grad denotes derivative with respect to $x=f\left(X_{d}, t\right)$ and $\mathbf{h}_{d}=J \mathbf{F}_{d}^{-1}(\mathbf{h} \circ f)$ and $\mathbf{D}_{d}=J \mathbf{F}^{-1}(\mathbf{D} \circ f) \mathbf{F}^{-T}$.
} 
The initial-boundary value problem describing the dynamics of the gel is the following: given the domain $\mathcal{B}_{d} \times \mathcal{T}$, find $\left(\mathbf{u}_{d}, c_{d}, p\right)$ such that the following bulk balance equations

$0=\operatorname{div} \mathbf{S}_{d} \quad$ and $\quad \dot{c}_{d}=-\operatorname{div} \mathbf{h}_{d}$ on $\mathcal{B}_{d} \times \mathcal{T}$,

mechanical boundary conditions

$\mathbf{S}_{d} \mathbf{m}=\mathbf{t}$ on $\partial_{t} \mathcal{B}_{d} \times \mathcal{T}$ and $\mathbf{u}_{d}=\overline{\mathbf{u}}_{d}$ on $\partial_{u} \mathcal{B}_{d} \times \mathcal{T}$,

chemical boundary conditions

$-\mathbf{h}_{d} \cdot \mathbf{m}=q$ on $\partial_{q} \mathcal{B}_{d} \times \mathcal{T}$ and $\mu=\mu_{e}$ on $\partial_{c} \mathcal{B}_{d} \times \mathcal{T}$,

volumetric constraint

$J_{d}=\hat{J}_{d}\left(c_{d}\right)$ on $\mathcal{B}_{d} \times \mathcal{T}$,

and initial conditions

$\mathbf{u}_{d}=\mathbf{u}_{o}, c_{d}=c_{o}$ and $p=p_{o}$ on $\mathcal{B}_{d} \times\{0\}$,

hold. In Eqs. 2.8 and 2.9, $\partial_{t} \mathcal{B}_{d}, \partial_{u} \mathcal{B}_{d}, \partial_{q} \mathcal{B}_{d}$, and $\partial_{c} \mathcal{B}_{d}$ represent the portion of the boundary where it is controlled the force $\mathbf{t}$, the displacement $\overline{\mathbf{u}}$, the solvent flux $q$, and the chemical potential $\mu_{e}$, respectively. Moreover, a dot denotes the time derivative, div the divergence operator, and $\mathbf{m}$ the outward unit normal to $\partial \mathcal{B}_{d}$. It is worth noting that the balance equations (2.7) constitute a system coupled by both the volumetric constraint (2.1) and the constitutive equations (2.2) and (2.3). As we assume both the bulk force and the bulk solvent source to be null, the state of the system is determined by the boundary conditions; in particular, solvent uptake or release, which takes place at the boundary, depends on the applied force $\mathbf{t}$ and on the external chemical potential $\mu_{e}$ [23]. The solvent volume contained in the gel at time $t$ is easily determined by

$V_{s}(t)=\int_{\mathcal{B}_{d}} \Omega c_{d}\left(X_{d}, t\right) d V_{d}, \quad\left[V_{s}\right]=\mathrm{m}^{3}$.

Here, we consider as main driving force of solvent dynamics the difference between $\mu_{e}$, and the chemical potential $\mu$ of the solvent within the polymer. Thus, Eq. 2.92, imposed at the boundary, may be interpreted as a boundary condition which relates the solvent concentration $c_{s}$, and the pressure $p$ at boundary to the value of $\mu_{e}$ :

$\hat{\mu}\left(c_{s}\right)+p \Omega=\mu_{e} \quad$ and $\quad c_{d}=c_{s}, \quad$ on $\quad \partial_{c} \mathcal{B}_{d} \times \mathcal{T}$.

It is worth noting that Eq. 2.13 is a nonlinear equation which cannot be solved explicitly for $c_{s}$; moreover, on $\partial_{c} \mathcal{B}_{d}$, where $c_{s}$ is controlled through Eq. $2.13_{1}$, the solvent flux $q$ is a reaction, unknown a priori. Typically, as standard for reactions, $q$ is evaluated during post-processing. It yields a poor approximation of the solvent flux, which is a relevant quantity in solvent dynamics. To overcome the issue, in the finite element implementation of the problem, we use the following integral versions of the boundary conditions controlling the solvent concentration [14, 27, 31]:

$0=\int_{\partial_{c} \mathcal{B}_{d}}\left[\hat{\mu}\left(c_{s}\right)+p \Omega-\mu_{e}\right] \cdot \tilde{c}_{s} d V_{d} \quad$ and $\quad 0=\int_{\partial_{c} \mathcal{B}_{d}}\left[\left(c_{d}-c_{s}\right) \tilde{q}+q\left(\tilde{c}_{d}-\tilde{c}_{s}\right)\right] d V_{d}$

where $\tilde{c}_{d}, \tilde{c}_{s}$, and $\tilde{q}$ are test functions of the finite element method. The first equation is the integral form of Eq. $2.13_{1}$. The second equation enforces the constraint $c_{d}=c_{s}$ by considering $q$ as an additional state variable, having the role of a Lagrange multiplier: it provides a better evaluation of $q$ and of other $q$-based quantities.

Finally, another important quantity is the overall time rate of solvent volume $\dot{Q}$ crossing the boundary per unit time and unit area, defined as

$\dot{Q}=\Omega \int_{\partial \mathcal{B}_{d}} q d A_{d}, \quad[\dot{Q}]=\mathrm{m}^{3} / s$

note that $q>0$ is an inward flux. By evaluating $\dot{Q}$ on the boundary of the cavity, it is possible to compute the time course of the solvent content in the cavity.

\section{Solvent release from a polymer with small solvent-filled cavities}

Here, we tackle the problem of solvent release from a gel with a small cavity and start from a fully swollen state with the cavity completely filled with solvent. Dynamics of solvent release when small solvent-filled cavities are present in the bulk 
polymer need further remarks. Being the solvent incompressible, the cavity volume must always be equal to the volume of the solvent it contains; thus, when solvent is pumped out of the cavity, the cavity volume reduces and the cavity wall is pulled by an increasing negative pressure which affects the chemical potential of the solvent inside the cavity. The consequence is that a steady, yet not homogeneous, state can take place. As is will be discussed in the following, the suction (negative) pressure is determined by different material conditions which make possible or not the existence of a steady state other than the initial one.

Let us consider a gel $\mathcal{B}_{d}$ and its cavity $\mathcal{C}_{d}$ at dry state. The corresponding swollen, steady, and stress-free state is $\mathcal{B}_{o}$ and $\mathcal{C}_{o}$ denotes the cavity in this state which has a size different from $\mathcal{C}_{d}$ 's size $[29,30]$. This swollen state is uniquely determined by the conditions $\mathbf{S}_{d}=\mathbf{0}$ and $\mu=\mu_{o}$. From Eq. 2.4, it follows a relation between the uniform swelling ratio $\lambda_{o}$ of the gel and $\mu_{o}$, with $\mu_{o}$ the value of the chemical potential of the solvent in the bath and in the cavity:

$\mathcal{R} T\left(\log \frac{\lambda_{o}^{3}-1}{\lambda_{o}^{3}}+\frac{1}{\lambda_{o}^{3}}+\frac{\chi}{\lambda_{o}^{6}}\right)+\frac{G}{\lambda_{o}} \Omega=\mu_{o}$.

The homogeneous state $\mathcal{B}_{o}$ is not equilibrated if a change in the chemical potential is assigned at the boundary. Let us distinguish between the boundary $\partial_{e} \mathcal{B}_{d}$ of the polymer gel body and the boundary $\partial_{i} \mathcal{B}_{d}$ of the cavity; we reserve the symbol $\mu_{e}$ for the chemical potential of the external bath whereas denote with $\mu_{i}$ the chemical potential of the solvent within the cavity. In general, if a chemical potential $\mu_{e}<\mu_{o}$ is assigned on $\partial_{e} \mathcal{B}_{d}$, diffusion starts and solvent is expelled from both the gel and the cavity until a new equilibrium state is attained (see Fig. 1).

In this investigation, we focus on the dynamics of solvent concentration in the gel under controlled changes of $\mu_{e}$. Dynamics is described by Eq. 2.7 and is driven by the changes in the chemical boundary conditions (see Eq. 2.9). We assume that (i) the cavity stays always filled with solvent, that is, an incompressible liquid and set $\mu_{i}=\Omega p_{i}(t)$ with the pressure term $p_{i}$ representing the suction pressure; (ii) the outer environment is filled with air, that is, an ideal gas whose content in water determines the value of the chemical potential which can be related to the relative humidity of the air, and

Fig. 1 We consider two polymer spheres immersed in a solvent, without (a) and with (b) a cavity completely filled with the same solvent; both spheres are in a free-swollen, steady state. When the spheres are removed from the bath (bottom row), the solvent is released from both the bulk polymeric matrix and the cavity. The key point for the sphere with the cavity is that the change of the solvent volume in the cavity yields a change of the pressure acting on the cavity wall, which plays an important role in the dynamics of solvent release. This inner pressure can become negative, and brings a stop in the dynamics; thus, also non homogenous steady states are possible, having different conditions on the outside boundary and in the inside one a)
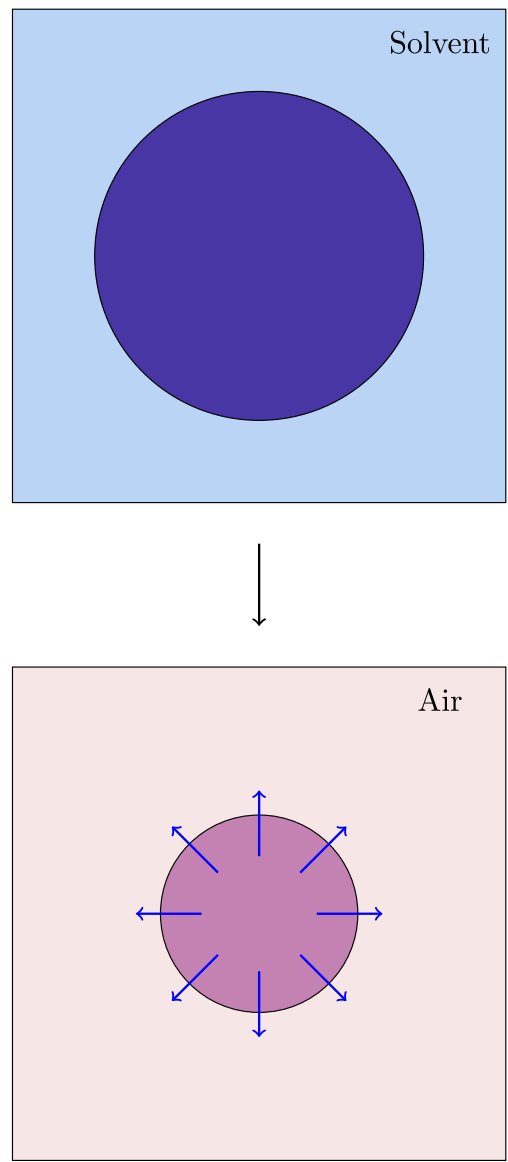

b)
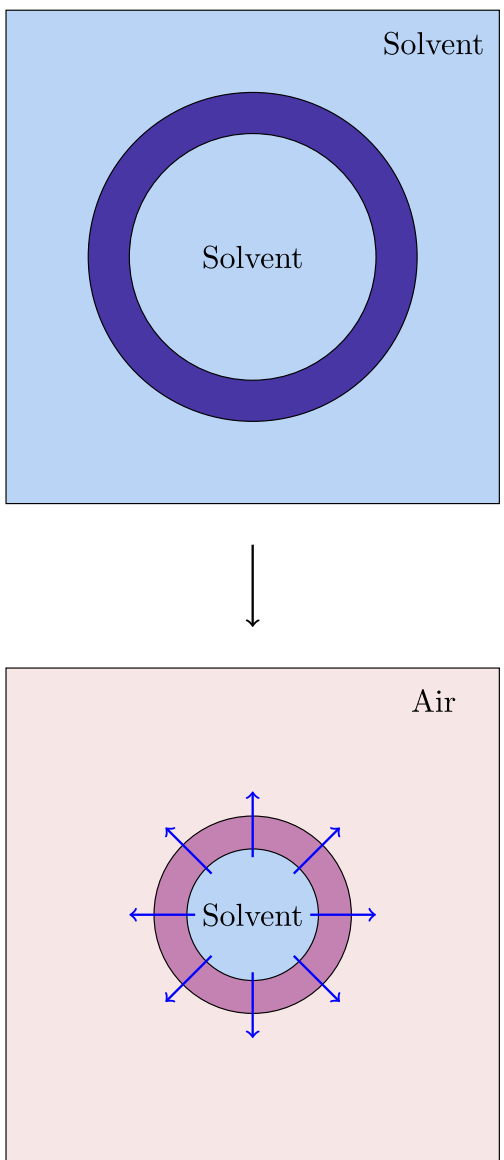
set $\mu_{e}=\hat{\mu}_{e}(t)$ with $\hat{\mu}_{e}(t)$ the (unique) control law of the problem (see cartoon in Fig. 3). So, in the end, we write down at any time $t \in \mathcal{T}$ :

$\mathbf{S}_{d} \mathbf{m}=-p_{e} \mathbf{F}_{d}^{\star} \mathbf{m}=\mathbf{0}$ and $\mu_{e}=\hat{\mu}_{e}(t)$ on $\partial_{e} \mathcal{B}_{d}$,

and

$\mathbf{S}_{d} \mathbf{m}=-p_{i} \mathbf{F}_{d}^{\star} \mathbf{m} \quad$ and $\quad \mu_{i}=\Omega p_{i}(t) \quad$ on $\quad \partial_{i} \mathcal{B}_{d}$,

where we neglected the atmospheric pressure $p_{e}$. Equations 3.16 and 3.17 show that a steady state is attained after a change of the external chemical potential from $\mu_{o}$ when $\mu_{i}$ attains the same value $\mu_{e}$. On the other hand, as in the dehydration process $\mu_{e}<0$, a negative pressure $p_{i}$ is required to get a steady state. The negative pressure $p_{i}=p_{i}(t)$ realizes the suction effect and is modelled as the reaction to the volumetric coupling between the volume $V_{s}^{c}=V_{s}^{c}(t)$ of the solvent in the cavity and the volume of the cavity $V_{c}=V_{c}(t)$,

$V_{c}(t)=V_{s}^{c}(t)$,

which must hold at each instant $t \in \mathcal{T}$ as solvent flows out of the cavity (see also [31]). It is worth noting that the global constraint (3.18) adds a further coupling between the state variables of the multiphysics problem other than the common local volumetric constraint (2.1). Constraint (3.18) can be enforced by considering the augmented total free energy defined by

$\int_{\mathcal{B}_{d}} \psi_{r} d V_{d}-p_{i}\left(V_{c}-V_{s}^{c}\right)$,

so that the cavity pressure $p_{i}$ can be viewed as the Lagrange multiplier enforcing the constraint. The cavity volume $V_{c}$ depends on the actual configuration $\mathcal{C}_{t}=f\left(\mathcal{C}_{d}, t\right)$ of the cavity $\mathcal{C}_{d}$ at time $t$, and can be measured by evaluating the following integral

$V_{c}(t)=\int_{\mathcal{C}_{t}} d v=-\frac{1}{3} \int_{\partial_{i} \mathcal{B}_{d}(t)} x \cdot \mathbf{n} d a=-\frac{1}{3} \int_{\partial_{i} \mathcal{B}_{d}}\left(X_{d}+\mathbf{u}_{d}\right) \cdot \mathbf{F}_{d}^{\star} \mathbf{m} d A_{d}$,

with $\mathbf{n}$ the normal to $\partial_{i} \mathcal{B}_{d}(t)=f\left(\partial_{i} \mathcal{B}_{d}, t\right) .{ }^{3}$ The solvent volume at time $t$ is the sum of the initial solvent content $V_{s}^{c}(0)$ of the cavity, plus the solvent volume $Q_{i}(t)$ the has crossed the cavity boundary during the time interval $(0, t)$, that is, $V_{c}^{s}(t)=V_{s}^{c}(0)+Q_{i}(t)$. The initial solvent content equals the initial cavity volume $V_{c}(0)$; thus, from Eq. 3.20 it follows:

$V_{s}^{c}(0)=V_{c}(0)=-\frac{1}{3} \int_{\partial_{i} \mathcal{B}_{d}}\left(X_{d}+\mathbf{u}_{o}\right) \cdot \mathbf{F}_{o}^{\star} \mathbf{m} d A_{d}$,

with $\mathbf{F}_{o}^{\star}=J_{o} \mathbf{F}_{o}^{-T}$ and $J_{o}$ the adjugate and the Jacobian determinant of the initial swollen deformation gradient $\mathbf{F}_{o}=\lambda_{o} \mathbf{I}$. The solvent volume $Q_{i}(t)$ that has crossed the cavity boundary entering into the gel in the time interval $(0, t)$ can be evaluated by the time integration of a formula analogous to Eq. 2.14; it holds

$Q_{i}(t)=\int_{0}^{t} \dot{Q}_{i}(\tau) d \tau=\Omega \int_{0}^{t} \int_{\partial_{i} \mathcal{B}_{d}} q d A_{d} d \tau=-\Omega \int_{0}^{t} \int_{\partial_{i} \mathcal{B}_{d}} \mathbf{h}_{d} \cdot \mathbf{m} d A_{d} d \tau$.

\section{Control of solvent release from spherical polymer capsules}

Let us consider a polymer sphere with a small sphere cavity, that is, a spherical capsule. Polymer-based microcapsules can form a covering to substances which have to be protected from modification or degradation before being released at the correct location [36-38] and can also be used in microfluidic systems where solvent release processes can be implemented to drive specific functional demands [39,40]. In both cases, the control of solvent release and of its rate is important and requires a study which combines the nonlinear and transient mechanics of the micro-system with the dynamics of the release process.

\footnotetext{
${ }^{3}$ We note that the internal boundary of the gel $\partial_{i} \mathcal{B}_{d}$ coincides with the boundary of the cavity $\partial \mathcal{C}_{d}$, proviso an opposite orientation of the normal.
} 


\subsection{Spherical dynamics and solvent release conditions}

The dry state of the polymer is a hollow sphere $\mathcal{B}_{d}$ with radius $r_{d}$, and its cavity is a smaller sphere $\mathcal{C}_{d}$ with radius $r_{c}$. From Eq. 3.15, it follows that the corresponding swollen state, assumed steady and stress free, is the sphere $\mathcal{B}_{o}$ with radius $\lambda_{o} r_{d}$; we denote with $\mathcal{C}_{o}$ the cavity at the swollen state with radius $\lambda_{o} r_{c}$. Taking $\mathcal{B}_{o}$ as the initial state, we change the chemical potential $\mu_{e}$ : diffusion starts, solvent is released from both the bulk and the cavity, and eventually, a new equilibrium state is attained.

This process has spherical symmetry, and the deformation of the body can be described as $x=f_{d}(r, t)=(r+u(r, t)) \mathbf{n}$, and $c_{d}=c_{d}(r, t)$, with $r$ the radial coordinate, $u$ the radial displacement, and $\mathbf{n}$ the unit radial vector. Under these assumptions, it holds

$\mathbf{F}_{d}=\lambda_{r} \mathbf{n} \otimes \mathbf{n}+\lambda_{\theta}(\mathbf{I}-\mathbf{n} \otimes \mathbf{n}) \quad$ with $\quad \lambda_{r}=f_{d}^{\prime}=1+u^{\prime} \quad$ and $\quad \lambda_{\theta}=\frac{f_{d}}{r}=1+\frac{u}{r}$,

being $\lambda_{r}$ and $\lambda_{\theta}$, the radial and hoop stretch, respectively, and a prime denoting derivation with respect to the radial coordinate $r$; from Eq. 4.23, it follows $J_{d}=\lambda_{r} \lambda_{\theta}^{2}$.

The Piola-Kirchhoff stress $\mathbf{S}_{d}$ has only the radial $\sigma_{r}$ and the circumferential $\sigma_{\theta}$ components different from zero:

$\sigma_{r}=G \lambda_{r}-p \lambda_{\theta}^{2} \quad$ and $\quad \sigma_{\theta}=G \lambda_{\theta}-p \lambda_{r} \lambda_{\theta}$.

The Cauchy stress $\mathbf{T}=\mathbf{S}_{d} \mathbf{F}^{T} / J_{d}$ has components:

$T_{r}=G \lambda_{r} / \lambda_{\theta}^{2}-p \quad$ and $\quad T_{\theta}=G / \lambda_{r}-p$.

The reference solvent flux is described by a single scalar field

$h_{d}=-\frac{D c_{d}}{R T} \lambda_{r}^{-2} \mu^{\prime}$,

and the corresponding component of the actual solvent flux $\mathbf{h}$ is $h=h_{d} / \lambda_{\theta}^{2}$. The global volume constraint (3.18) rewrites considering that

$$
\begin{aligned}
V_{c}(t) & =\frac{4}{3} \pi r(t)^{3}=\frac{4}{3} \pi\left(r_{c}+u\left(r_{c}, t\right)\right)^{3}=\frac{4}{3} \pi r_{c}^{3} \lambda_{\theta}\left(r_{c}, t\right)^{3}, \\
\dot{Q}_{i}(t) & =\Omega 4 \pi r_{c}^{2}\left(-\frac{D c_{d}\left(r_{c}, t\right)}{R T}\left(1+u^{\prime}\left(r_{c}, t\right)\right)^{-2} \mu^{\prime}\left(r_{c}, t\right)\right), \\
V_{s}^{c}(0) & =\frac{4}{3} \pi\left(\lambda_{o} r_{c}\right)^{3} .
\end{aligned}
$$

The initial-boundary value problem describing the dynamics of the spherical gel is the following: given the domain $\left(r_{c}, r_{d}\right) \times \mathcal{T}$, find $\left(u, c_{d}, p, p_{i}\right)$ such that, at any time $t \in \mathcal{T}$, the bulk balance equations

$\sigma_{r}^{\prime}+\frac{2}{r}\left(\sigma_{r}-\sigma_{\theta}\right)=0 \quad$ and $\quad \dot{c}_{d}=-\left(h_{r}^{\prime}+\frac{2}{r} h_{r}\right)$ for $\quad r \in\left(r_{c}, r_{d}\right)$,

the external boundary conditions

$\sigma_{r}=0 \quad$ and $\quad \mu=\mu_{e}$ at $r=r_{d}$,

the internal boundary conditions

$\sigma_{r}=-\lambda_{\theta}^{2} p_{i} \quad$ and $\quad \mu=\Omega p_{i}$ at $r=r_{c}$,

the volumetric constraint

$J_{d}=\lambda_{r} \lambda_{\theta}^{2}=\hat{J}_{d}\left(c_{d}\right)$ for $r \in\left(r_{c}, r_{d}\right)$,

the global volumetric constraint

$V_{c}(t)=V_{s}^{c}(0)+Q_{i}(t)$,

the initial conditions

$u=\left(\lambda_{o}-1\right) r, c_{d}=\left(\lambda_{o}^{3}-1\right) / \Omega \quad$ and $\quad p=p_{o}$ for $r \in\left(r_{c}, r_{d}\right)$ and $t=0$,

hold. 



Fig. 2 Pressure-volume curves with $\chi=0.2, T=293 \mathrm{~K}, \Omega=1.8 \cdot 10^{-5} \mathrm{~m}^{3} / \mathrm{mol}$, external radius $r_{d}=1 \mathrm{~cm}$, cavity radius $r_{c}=0.9 \mathrm{~cm}$ and $D=10^{-9} \mathrm{~m}^{2} / \mathrm{s}$. Orange background denotes the range of cavity volume where pressure is positive, while pink denotes the region with negative pressure. The panels correspond to poorly swollen gels $\left(\varepsilon=3 \cdot 10^{-1}, \lambda_{o}=1.15\right.$, panel (a)) and highly swollen gels $\left(\varepsilon=3 \cdot 10^{-3}, \lambda_{o}=2.45\right.$, panel (b)). a Pressure takes negative values at $V_{c}(t) / V_{c}(0) \approx 0.8$ and, depending on the value of $\mu_{e}$, equilibrium states are theoretically achievable from that cavity volume ratio. $\mathbf{b}$ A high positive pressure region occurs because the cavity gets compressed by the walls of the microcapsule which shrink because solvent exits from them; in this case pressure becomes negative at $V_{c}(t) / V_{c}(0) \approx 0.15$

We assign a change in the external chemical potential $\mu_{e}=\hat{\mu}_{e}(t)$, with $\hat{\mu}_{e}(t)$ a step-wise constant function. A step change of $\hat{\mu}_{e}(t)$ yields a transient solvent release which stops when a new equilibrium state is attained; a further step change of the external chemical potential produces a new release until another steady state is reached. It turns out that by tuning $\hat{\mu}_{e}(t)$, it is possible to control both the duration release and the amount of solvent exiting from the microcapsule.

\subsubsection{Highly and poorly swollen gels}

When a polymer body has small cavities and solvent can be released from both the cavity and the bulk, it is especially important to distinguish between highly and poorly swollen gels as it can determine a very different dynamics. Equation 3.15 is the right tool to do it. Indeed, the swelling ratio $\lambda_{o}$ corresponding to $\mu_{o}$ which makes the difference between a highly and a poorly swollen gel depends on the shear modulus $G$ and the parameter $\chi$ which describes the polymer-solvent affinity. Let us introduce the dimensionless parameter $\epsilon=G \Omega / R T$, which is the ratio between the two key material constants of the mechanical and chemical free energy. ${ }^{4}$ Then, we define a regime of poorly swollen gels when:

$\epsilon \gtrsim 10^{-1}$ and $\chi \lesssim 0.8$ or $\epsilon \lesssim 10^{-1}$ and $\chi \gtrsim 0.8$.

In this case, Eq. 3.15 yields $\lambda_{o} \lesssim 1.5$. On the other hand, we define a second regime of highly swollen gels when:

$\epsilon \lesssim 10^{-1}$ and $\chi \lesssim 0.8$.

In this case, Eq. 3.15 yields $\lambda_{o} \gtrsim 2$. For highly swollen gels, due to the great amount of water inside microcapsule walls, solvent is firstly released from the gel rather than from the cavity. As a consequence, suction effect does not become apparent and the inner pressure $p_{i}$ takes non-negative values (see Fig. 2, panel (b)). Under these conditions, the new equilibrium state is attained at very low ratios $V_{c}(t) / V_{c}(0) \lesssim 0.1$.

By contrast, for poorly swollen gels, solvent is mainly released from the cavity and the inner pressure takes negative values. Under these conditions, the new equilibrium state is attained at higher ratios $V_{c}(t) / V_{c}(0)$ with respect to the former case (see Fig. 2, panel (a)).

It is worth noting that the suction effect induced by a negative inner pressure can produce mechanical instabilities and buckling phenomena; the study of these phenomena is beyond the scope of the present work.

\subsection{Poorly swollen gel microcapsules}

We limit our analysis to problems where the thermodynamical equilibrium is attained for $V_{c}(t) / V_{c}(0)>0.3$ and the conditions corresponding to the first regime hold. The aim is producing a stop-and-go mechanism of solvent fluxes from microcapsules through the accurate tuning of the external chemical potential $\mu_{e}$.

\footnotetext{
${ }^{4}$ Indeed, the ratio $R T / / \Omega$ has the same role for the chemical energy as that of the shear modulus $G$ for the elastic energy.
} 
We consider gels whose material and geometrical characteristics are $G=50 \mathrm{MPa}, \chi=0.2$, external dry radius $r_{d}=1$ cm, cavity dry radius $r_{c}=0.9 \mathrm{~cm}$, molar volume of the solvent $\Omega=1.8 \cdot 10^{-5} \mathrm{~m}^{3} / \mathrm{mol}$ and diffusivity $D=10^{-9}$ $\mathrm{m}^{2} / \mathrm{s}$; we also set $T=293 \mathrm{~K}$. With these values, the initial solvent content in the microcapsules's walls is $V_{s}(0)=0.60$ $\mathrm{cm}^{3}, \varepsilon=0.37$, and the initial swelling ratio is $\lambda_{o}=1.1525$. The dynamics described by the Eq. $2.7_{2}$ introduces the characteristic diffusion time $\tau_{d}=l^{2} / D \varepsilon$ into the model, where $l=r_{d}-r_{c}$ is the characteristic diffusion length; it holds $\tau_{d}=\left(r_{d}-r_{c}\right)^{2} / D \varepsilon \simeq 10^{5} \mathrm{~s}$.

We assume that the external chemical potential $\mu_{e}$ changes with the step-wise constant law $\hat{\mu}_{e}(t)$, having a step decrease $\Delta \mu_{e}=-200 \mathrm{~J} / \mathrm{mol}$ after every time interval $\Delta t=4 \cdot 10^{5} \mathrm{~s}$; for comparison, we also study dynamics due to a single step $\Delta \mu_{e}=-1 e 3 \mathrm{~J} / \mathrm{mol}$. We start from the initial value $\mu_{e}=0 \mathrm{~J} / \mathrm{mol}$ and stop at the final value $\mu_{e}=-1 e 3 \mathrm{~J} / \mathrm{mol}$, corresponding for example, if humidity environment is controlled, to a relative humidity $R H=66.3 \%$.

In Fig. 3 (top panels), we show a cartoon of the initial (left), intermediate (center), and final (right) state of the microcapsule. In the same figure (middle left panel), we show the time laws $\hat{\mu}_{e}(t)$ corresponding to a single-step (green, dashed) and a step-wise constant function (blue, solid). The same color code is used to represent the corresponding dynamics represented through the time evolution of a few key elements obtained by solving the coupled stress-diffusion problem. The pressure inside the cavity $p_{i}$ (middle right panel) corresponding to the two different control laws of $\hat{\mu}_{e}(t)$ is represented. It is always negative and yields an inner chemical potential which contributes to reducing the gradient of the chemical potential across the wall thickness. Finally, an equilibrium steady state is attained when the inner pressure takes a specific value which makes null the gradient of the chemical potential. The stop-and-go solvent flux is also shown (bottom left panel); as expected, solvent flux presents a peak at each time interval $\Delta t$ and solvent peaks decrease with time. The relation between flux and external chemical potential is nonlinear and it is qualitatively similar to the relation between the uniform swelling ratio $\lambda_{o}$ and the external chemical potential in Eq. 3.15. Finally, the ratio between the solvent volume $Q_{e}$ expelled from the capsule and the total initial solvent content $V_{s}^{T}(0)$ is also shown (bottom right panel) with:

$\dot{Q}_{e}=\Omega \int_{\partial_{e} \mathcal{B}_{d}} q d A_{d}, \quad$ and $\quad V_{s}^{T}(t)=V_{s}(t)+V_{s}^{c}(t)$.

Fig. 3 Dynamics of solvent release from a hollow spherical gel. Top. Cartoons of the initial (left) and final (right) equilibrium state of the microcapsule; a sketch of a far from thermodynamical equilibrium state is also represented (center). Middle, left: Time evolution of $\mu_{e}$ for a single step (dashed green line) and for multiple steps (solid blue line) (left); the same color code is used to represent the corresponding dynamics. The remaining three panels show the key outcome of the model: the pressure inside the cavity $p_{i}$ (middle, right), which has negative values; (bottom, left), the actual flux from the external walls which stops and starts at each time interval $\Delta t$; the ratio between the solvent volume $Q_{e}$ expelled from the capsule and the total initial solvent content $V_{s}^{T}(0)$ (bottom, right)


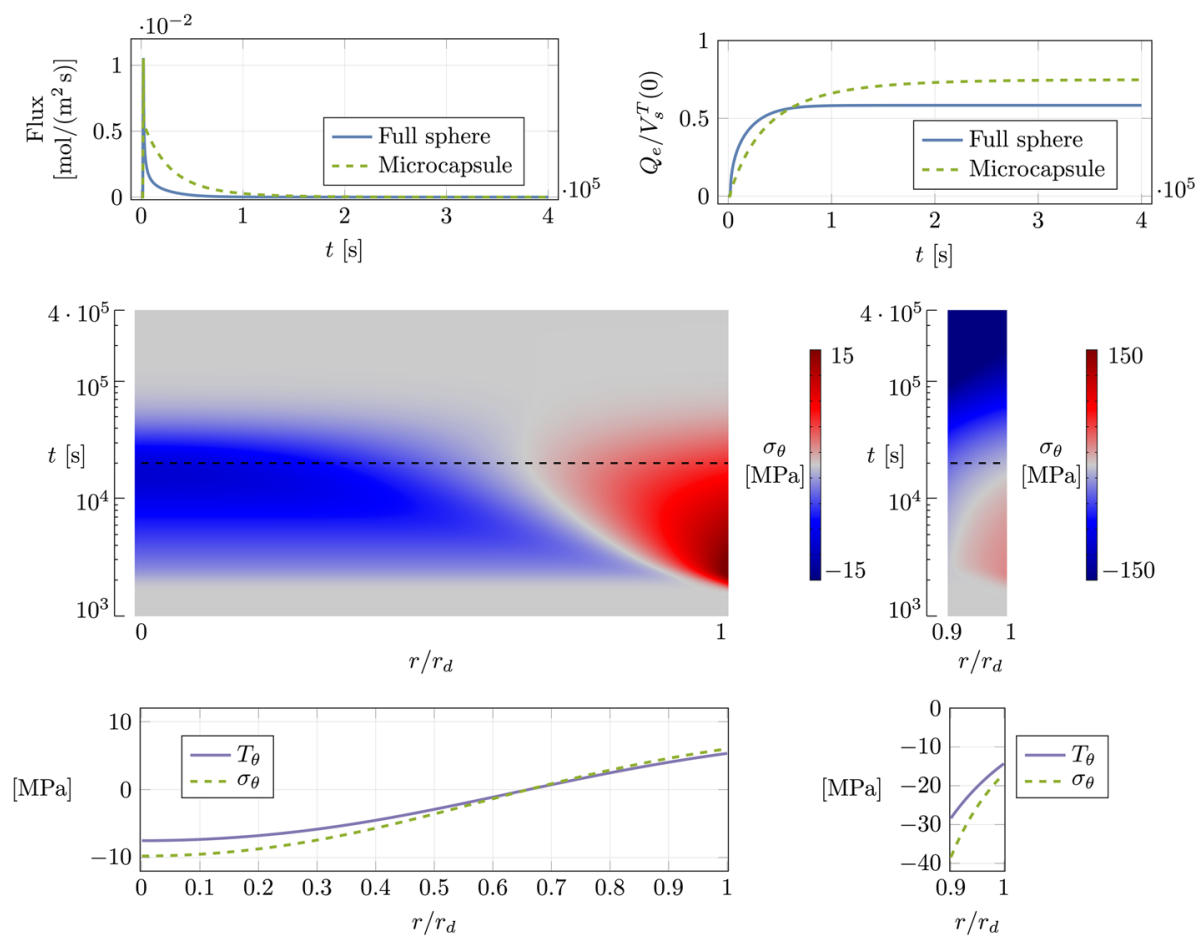

Fig. 4 Comparison between a spherical and a hollow spherical gel. Top. Actual solvent flux versus time for the full sphere (solid) and the microcapsule (dashed) corresponding to the single-step law for $\mu_{e}$, see Fig. 3 (left); ratio between the solvent volume $Q_{e}$ expelled and the initial solvent content $V_{s}^{T}(0)$ versus time for the full sphere (solid) and the microcapsule (dashed). Middle. Contour plot of the hoop stress $\sigma_{\theta}$ versus the radius $r$ and time in the full sphere (left) and in the microcapsule (right). It is worth noting that the hoop stress in the microcapsule is tenfold than the one in the sphere; moreover, at steady state, the sphere becomes stress free (gray color at $t_{\infty}$ ), while the microcapsule remains highly compressed (blue color at $\left.t_{\infty}\right)$. Bottom. Piola-Kirchhoff and Cauchy hoop stresses are compared at the same instant $t=2 \cdot 10^{4} \mathrm{~s}($ dashed black line in the middle panel)

Precisely, $Q_{e}$ is the solvent volume crossing the external boundary, and comprehend both the solvent initially in microcapsule's walls and inside the cavity; $V_{s}^{T}(0)$ is the volume of solvent initially in the gel walls and within the cavity; this latter corresponds, as already written, to the initial volume $V_{c}(0)$ of the cavity.

\subsection{Hollow sphere versus sphere}

We compare the solvent release from a sphere of radius $r_{d}$ and a hollow sphere having the same external radius $r_{d}$, with a spherical cavity of radius $r_{c}$ in terms of fluxes, ratio between the solvent volume expelled from the two polymer structures and the total initial solvent content and stresses. Firstly, we evaluate the total solvent content $V_{s}^{T}(0)$ of the sphere which corresponds to the solvent $V_{S}(0)$ in its bulk and is determined by the initial value $\mu_{o}$ of the chemical potential:

$V_{s}^{T}(0)=V_{s}(0)=\frac{4}{3} \pi r_{d}^{3}\left(\lambda_{o}^{3}-1\right)$

The analogous quantity $V_{s}^{T}(0)$ for the hollow sphere is given by:

$V_{s}^{T}(0)=V_{s}(0)+V_{s}^{c}(0)=\frac{4}{3} \pi r_{d}^{3}\left(\lambda_{o}^{3}-1\right)+\frac{4}{3} \pi r_{c}^{3}$.

Thus, under the same conditions, the hollow sphere contains more solvent, and the difference between the two is given by the volume of the dry cavity $4 \pi r_{c}^{3} / 3$. The differences between the two polymer structures are not limited to that there are a few key aspects of the dynamics during the solvent release which make the two situation quite different. 
The volume of solvent released in the time interval $(0, t)$ from both a full sphere and a microcapsule due to a change in the chemical potential $\mu_{e}$ is always measured by its rate $\dot{Q}_{e}$. Under the single-step decrease of the external chemical potential $\mu_{e}$, corresponding to the single-step control law shown in Fig. 3 (top left panel), the time evolution of the solvent actual fluxes through the external boundary has the pattern shown in Fig. 4 (top left panel: solid line for the sphere, dashed line for the hollow sphere). Our result shows that the full sphere releases solvent with a higher flux peak with respect to the hollow one, which releases the solvent more smoothly. Integrating those lines, the ratio between the amount $Q_{e}$ of released solvent normalized with respect to the initial total solvent content $V_{s}^{T}(0)$ can be obtained. Figure 4 (right panel) shows as the hollow sphere releases more solvent than the full one, under the same condition of external chemical potential.

An important difference between the two release systems is the stress state inside the polymer network. As expected, it is quite different all along the process. Figure 4 (middle panels) shows as hoop stresses are 1 order of magnitude higher in the microcapsule than in the full sphere and different from zero at the end of the process. Hence, whereas the full sphere is stress free at the steady state, the microcapsule presents a not uniform along the radius negative (compression) hoop stress. Finally, the hoop components of Piola-Kirchhoff and Cauchy stresses are compared in the bottom panel of Fig. 4, dashed green and purple solid line respectively. The qualitative behavior of the Piola-Kirchhoff and Cauchy stresses for the full sphere and capsule remains the same for all the dynamics.

\section{Conclusions and future perspectives}

A study which combines the nonlinear and transient mechanics of polymer-based structures with the dynamics of the release process from them has been presented. Attention has been restricted to polymers which release solvent from bulk and from small cavities. The dynamics of these systems is restricted by a global volumetric constraint which forces the solvent volume inside the cavity and the cavity geometric volume to match all along the process; the inner pressure on the cavity walls which maintains the constraint evolves in time, taking also negative values under some geometric and material conditions, until a steady state is attained.

We showed as the control of solvent release from the spherical capsule is achievable through smart changes of the outer chemical potential. During the dynamics of the process, high-stress states can be attained and maintained also at steady states. We also showed as steady states are attainable only for poorly swollen gels while highly swollen gels reach very high positive pressure which makes steady states in the reality unrealizable.

In our opinion, the present work suggests new lines of investigations based on the present analysis adapted for microcapsules and microtubules made of active polymer networks such as intelligent actively-remodeling biopolymer gels which can be used for controlled drug release [41] and actively-contractile photo-sensitive elastomers [42]. In these cases, a localized activation stimulus can deliver very different dynamics and produces smart actuation/delivery systems.

Funding Open access funding provided by Università degli Studi di Roma La Sapienza within the CRUI-CARE Agreement. The authors would like to thank MIUR (Italian Minister for Education, Research, and University) and the PRIN 2017, Mathematics of active materials: From mechanobiology to smart devices, project n. 2017KL4EF3, for financial support.

\section{Compliance with ethical standards}

Conflict of interest The authors declare that they have no conflict of interest.

Open Access This article is licensed under a Creative Commons Attribution 4.0 International License, which permits use, sharing, adaptation, distribution and reproduction in any medium or format, as long as you give appropriate credit to the original author(s) and the source, provide a link to the Creative Commons licence, and indicate if changes were made. The images or other third party material in this article are included in the article's Creative Commons licence, unless indicated otherwise in a credit line to the material. If material is not included in the article's Creative Commons licence and your intended use is not permitted by statutory regulation or exceeds the permitted use, you will need to obtain permission directly from the copyright holder. To view a copy of this licence, visit http://creativecommonshorg/licenses/by/4.0/.

\section{Appendix A: Cylindrical microtubules}

The same approach developed for spherical microcapsule hold when the confined cavities have cylindrical shape. For example, we consider a cylindrical infinitely long tubule having the same physical and geometrical parameters used for the microcapsule in the paper. Under free-swelling conditions, the microtubule gets a radius $\lambda_{o} r_{d}$ with $\lambda_{o}$ given by the Eq. 3.15 and $\mu_{e}=\mu_{o}$. The swollen tubule is the initial state of the our dynamic problem. Likewise we did for the spherical 

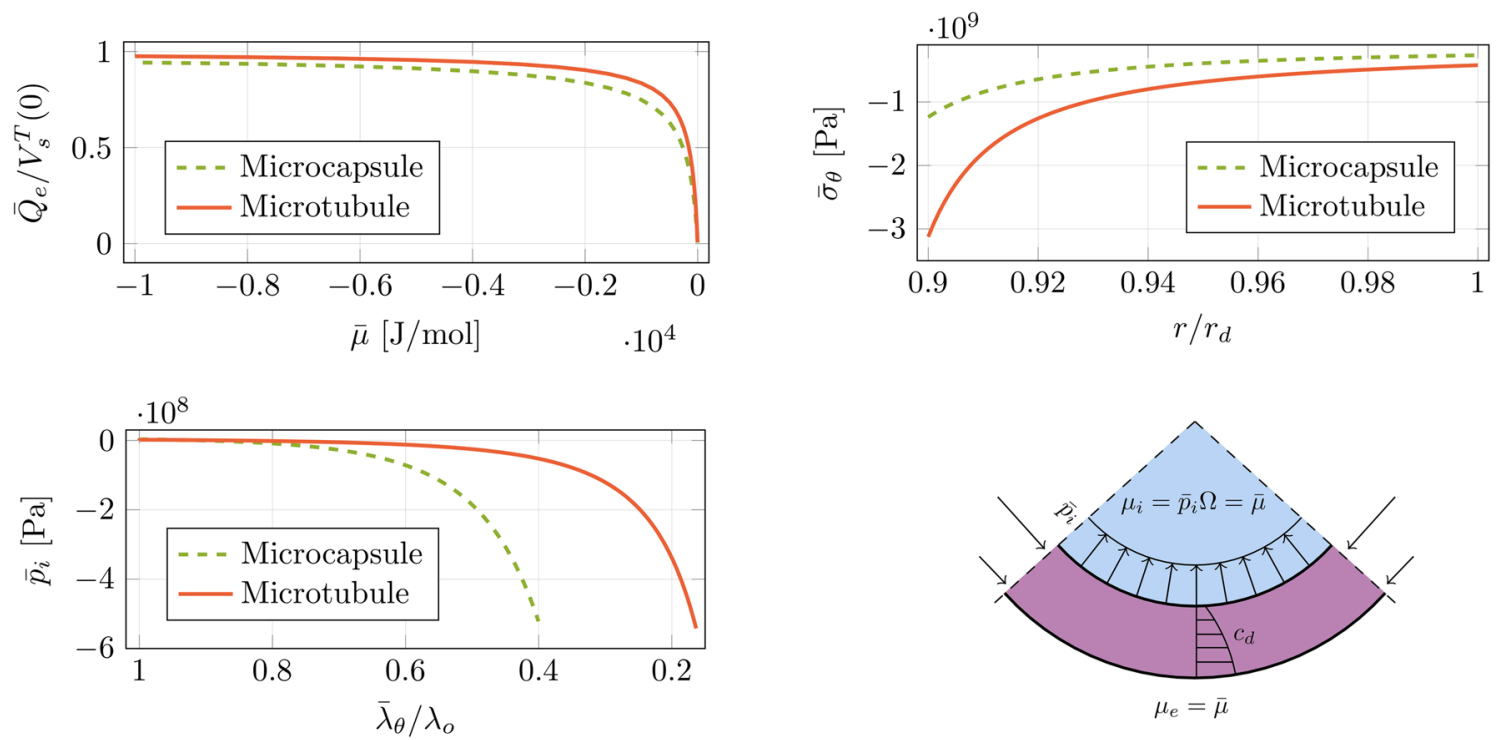

Fig. 5 Top. Ratio $Q_{e} / V_{s}^{T}(0)$ versus $\bar{\mu}$ in the microcapsule (dashed line) and in the microtubule (solid line) (left); hoop stress $\sigma_{\theta}$ versus dimensionless radius in the two systems. Bottom. Inflation curves $p_{i}$ versus $\lambda_{\theta} / \lambda_{o}$ in the microcapsule (dashed line) and in the microtubules (solid line) (left); cartoon representing the load condition of a part of the microtubule (right)

capsule, a reduction of the chemical potential $\mu_{e}$ starts the stress-diffusion mechanism and the solvent is released from both the bulk and the cavity, until a new steady state is attained. Due to the infinite length of the tubule, attention is restricted to the radially symmetric plane strain deformation of the cylindrical tubular region described by a deformation map $f_{d}(r, t)=(r+u(r, t)) \mathbf{n}$, with $r$ the radial coordinate, $u$ the radial displacement, and $\mathbf{n}$ the unit radial vector. Moreover, it is also assumed that $c_{d}=c_{d}(r, t)$. Under these assumptions, it holds:

$\mathbf{F}_{d}=\lambda_{r} \mathbf{e}_{r} \otimes \mathbf{e}_{r}+\lambda_{\theta} \mathbf{e}_{\theta} \otimes \mathbf{e}_{\theta}+\lambda_{z} \mathbf{e}_{3} \otimes \mathbf{e}_{3}$,

with $\lambda_{z}=\lambda_{o}$ and $\lambda_{r}$ and $\lambda_{\theta}$ the same as for the sphere. Under these conditions, the volume change ${ }^{5}$ of the tubule is $J_{d}=\lambda_{r} \lambda_{\theta} \lambda_{o}$; plane stresses and flux are:

$\sigma_{r}=G_{d} \lambda_{r}-p \lambda_{\theta} \lambda_{o}, \quad \sigma_{\theta}=G_{d} \lambda_{\theta}-p \lambda_{r} \lambda_{o}, \quad h_{d}=-\frac{D c_{d}}{R T} \lambda_{r}^{-2} \mu^{\prime}$,

whereas $\sigma_{z}=G_{d} \lambda_{z}-p \lambda_{r} \lambda_{\theta}$. The balance laws of forces and solvent are

$\sigma_{r}^{\prime}+\frac{1}{r}\left(\sigma_{r}-\sigma_{\theta}\right)=0 \quad$ and $\quad \dot{c}_{d}=-\left(h_{r}^{\prime}+\frac{1}{r} h_{r}\right)$.

The global volume constraint (3.18) rewrites considering that the tubule has infinite length and it can be written as a global area constraint in terms of the cavity cross-sectional area $A_{c}(t)$ and of the amount of solvent $A_{s}(t)$ in the cavity with

$A_{c}(t)=\pi r_{c}\left(r_{c}+u\left(r_{c}, t\right)\right) \lambda_{\theta}\left(r_{c}, t\right)$

$A_{s}(t)=A_{s}(0)-Q_{i}(t)$ and $\dot{Q}_{i}(t)=-2 \pi r_{c} \Omega h\left(r_{c}, t\right)$,

and $A_{s}(0)=A_{c}(0)$. The initial total amount of solvent is

$V_{s}^{T}(0)=\pi r_{c}^{2} \lambda_{o}^{2}+\pi\left(r_{d}^{2}-r_{c}^{2}\right)\left(\lambda_{o}^{3}-1\right)$.

The characteristic quantities of the release process are, as for the microcapsules, the ratio of the volume $Q_{e}$ of solvent crossing the external boundary over the initial total solvent content $V_{s}^{T}(0)$, the inner pressure $p_{i}$ and the hoop stress $\sigma_{\theta}$. We compare the values $\bar{Q}_{e}, \bar{p}_{i}$ and $\bar{\sigma}_{\theta}$ taken by these quantities at the equilibrium states corresponding to the constant value $\bar{\mu}$ of the external chemical potential $\mu_{e}$, controlled as a single-step function, in the microtubule and in the microcapsule. Figure 5 (top left panel) shows as the ratio $\bar{Q}_{e} / V_{s}^{T}(0)$ in the microcapsule (dashed) and in the microtubule (solid) is almost the

\footnotetext{
${ }^{5}$ Actually, under the hypothesis of plane strain, the axial stretch $\lambda_{z}$ remains constant and equal to $\lambda_{o}$; thus, $J_{d}$ measures the change in area of the cross-section
} 
same but in general microtubules release more solvent over the initial total content at the same change of external chemical potential. Each point of the two lines corresponds to the equilibrium state determined by the corresponding value $\bar{\mu}$ of the external chemical potential $\mu_{e}$. It is worth noting that for $\bar{\mu}<-2 \cdot 10^{3} \mathrm{~J} / \mathrm{mol}$, microcapsules may exhibit mechanical instabilities and buckling phenomena which can't be caught by solving the equations under the assumption of spherical symmetry. Also the hoop stress $\bar{\sigma}_{\theta}$ takes the comparable values shown in Fig. 5 (top right panel) in the two systems. On the other side, the inflation curves corresponding to the inner pressure $\bar{p}_{i}$ versus the hoop deformation $\bar{\lambda}_{\theta} / \lambda_{o}$ measured from the initial state are different as shown in Fig. 5 (bottom left panel) being the microcapsule (dashed line) stiffer than the microtubules (solid line). Finally, load conditions of the microtubule are shown in Fig. 5 (bottom right panel): the inner negative pressure and the compressive hoop stresses decreasing from the outer towards the center of the cross-section are shown.

\section{Appendix B: Integral formulations in symmetrical regions}

In the study of solvent release from gel (full or hollow) spheres and cylinders, due to geometrical and mechanical symmetries, we implemented in the finite element code the weak form of the equations of the multiphysics problem in a form reduced by considerations related to the symmetries. The time steps taken by the solver to compute the solution are chosen with a logarithmic entry method considering 20 steps per decade. Quadratic Lagrange shape functions are used for all state variables, while a Linear Lagrange shape function is used for the pressure $p$. The mesh size is very small: $1 / 1000$ the value of the external radius $r_{d}$.

In the following, the weak forms of the equations of both balance of forces and balance of solvent are summed up in the case of spherical symmetry. In the paper, the same equations have been developed in the case of cylindrical symmetry in a similar way.

When the equations have to be solved in a three-dimensional sphere-like region $\mathcal{B}_{d} \subset \mathcal{E}$, they are written down in terms of the spherical coordinates $(r, \theta, \psi)$ and the points $X \in \mathcal{B}_{d}$ are identified as

$X=o+r \mathbf{n}(\theta, \phi) \quad$ with $\quad \mathbf{n}(\theta, \phi)=\cos \phi \mathbf{i}_{3}+\sin \phi \mathbf{e}(\theta)$

and $\mathbf{e}(\theta)=\cos \theta \mathbf{i}_{1}+\sin \theta \mathbf{i}_{2}$ with $\theta \in(0, \pi / 2), \phi \in(0, \pi / 2)$, and $r \in\left(0, r_{d}\right)$ for a full sphere and $r \in\left(r_{c}, r_{d}\right)$ for a sphere with a spherical cavity of radius $r_{c}$.

The actual volume element $d v$ is related to the reference volume element $d V_{d}$ by

$d v(r, t)=J_{d}(r, t) d V_{d}=\lambda_{r}(r, t) \lambda_{\theta}^{2}(r, t) d V_{d}$

\section{Diffusion-type problem}

The prototype diffusion problem is governed by the following equation

$-\operatorname{div} \mathbf{h}_{d}=\dot{c}_{d} \quad$ in $\quad \mathcal{B}_{d} \quad$ and $\quad \mathbf{h}_{d} \cdot \mathbf{n}=q_{s} \quad$ on $\quad \partial \mathcal{B}_{d}$,

and solving it in the form reduced by the spherically symmetry means: (i) assuming that

$\mathbf{h}_{d}=h_{d}(r) \mathbf{n}(\theta, \phi) \quad$ and $\quad \operatorname{div} \mathbf{h}_{d}=\mathbf{I} \cdot \nabla \mathbf{h}_{d}=h_{d}^{\prime}(r)+\frac{2}{r} h_{d}(r) ;$

(ii) solving the local problem

$h_{d}^{\prime}(r)+\frac{2}{r} h_{d}(r)=-\dot{c}_{d} \quad$ in $\quad 0<r<R \quad$ and $\quad h_{d}=q_{s} \quad$ on $\quad r=r_{d}$,

when the region $\mathcal{B}_{d}$ is a full sphere and initial conditions have to be considered. Solving the same problem in a weak form means solving, for any test field $w$, the problem

$\int_{\mathcal{B}_{d}} \dot{c}_{d} w d V=-\int_{\mathcal{B}_{d}}\left(h^{\prime}+\frac{2}{r} h_{d}\right) w d V+\int_{\partial \mathcal{B}_{d}}\left(h_{d}\left(r_{d}\right)-q_{s}\left(r_{d}\right)\right) w\left(r_{d}\right) d A$.

As $d V=4 \pi r^{2} d r$ and $A=4 \pi R^{2}$, the Eq. 5.50 can be written down as

$0=\int_{0}^{R}\left(h_{d}^{\prime}+\frac{2}{r} h_{d}\right) w 4 \pi r^{2} d r-4 \pi R^{2}\left(h_{d}\left(r_{d}\right)-q_{s}\right) w\left(r_{d}\right)$. 
After some manipulations, we get the weak form corresponding to the spherically symmetric problem (5.49) which have to be implemented in the finite element code:

$\int_{0}^{r_{d}} \dot{c}_{d} w 4 \pi r^{2} d r=\int_{0}^{r_{d}}\left(h w^{\prime} 4 \pi r^{2} d r-4 \pi r_{d}^{2} q_{s} w\left(r_{d}\right)\right.$

The weak formulation for the hollow sphere reads the same for the bulk part of the equations while is different for what regards the contributes on the boundaries:

$\int_{\mathcal{B}_{d}} \dot{c}_{d} w d V=-\int_{\mathcal{B}_{d}}\left(h_{d}^{\prime}+\frac{2}{r} h_{d}\right) w d V+\int_{\partial \mathcal{B}_{d}}\left(h_{d}\left(r_{d}\right)-q_{e}\right) w\left(r_{d}\right) d A-\int_{\partial \mathcal{C}_{d}}\left(h_{d}\left(r_{c}\right)-q_{i}\right) w\left(r_{c}\right) d A$

where $\partial \mathcal{C}_{d}$ is the boundary of the spherical cavity $\mathcal{C}_{d} \subset \mathcal{B}_{d}$. With this, the final weak equation is

$\int_{r_{c}}^{r_{d}} \dot{c}_{d} w 4 \pi r^{2} d r=\int_{0}^{r_{d}} h_{d} w^{\prime} 4 \pi r^{2} d r-4 \pi r_{d}^{2} q_{e} w\left(r_{d}\right)+4 \pi r_{c}^{2} q_{i} w\left(r_{c}\right)$.

\section{Balance of forces-type problem}

The prototype balance-of-forces problem is governed by the following equation

$\operatorname{div} \mathbf{S}_{d}=0$ in $\mathcal{B}_{d} \quad$ and $\quad \mathbf{S}_{d} \mathbf{n}=\mathbf{t}$ on $\partial \mathcal{B}_{d}$

Solving the spherically symmetric problem in the sphere $\mathcal{B}_{d}$ means: (i) assuming that

$\mathbf{S}_{d}=\sigma_{r} \mathbf{n} \otimes \mathbf{n}+\sigma_{\theta} \mathbf{e}^{\prime} \otimes \mathbf{e}^{\prime}+\sigma_{\phi} \mathbf{n},_{\phi} \otimes \mathbf{n}, \phi$,

with all the three stress components only depending on $r$; (ii) solving the radial balance equation of forces as

$0=\operatorname{div} \mathbf{S}_{d} \cdot \mathbf{n}=\operatorname{div}\left(\mathbf{S}_{d}^{T} \mathbf{n}\right)-\mathbf{S}_{d} \cdot \nabla \mathbf{n}=\sigma_{r}^{\prime}+\frac{2}{r}\left(\sigma_{r}-\sigma_{\theta}\right) \quad$ in $\quad 0<r<r_{d}$,

and

$\sigma_{r}=\sigma \quad$ on $\quad r=r_{d}$.

Solving it in a weak form means solving, for any test field $w$, the problem

$0=\int_{\mathcal{B}_{d}}\left(\sigma_{r}^{\prime}+\frac{2}{r}\left(\sigma_{r}-\sigma_{\theta}\right)\right) w d V-\int_{\partial \mathcal{B}_{d}}\left(\sigma_{r}\left(r_{d}\right)-\sigma\right) w\left(r_{d}\right) d A$

that is, the problem

$0=\int_{0}^{r_{d}}\left(\sigma_{r}^{\prime}+\frac{2}{r}\left(\sigma_{r}-\sigma_{\theta}\right)\right) w 4 \pi r^{2} d r-4 \pi r_{d}^{2}\left(\sigma_{r}\left(r_{d}\right)-\sigma\right) w\left(r_{d}\right)$

After some manipulations, we get the weak form corresponding to the problem (5.57) and (5.58):

$0=-\int_{0}^{r_{d}}\left(\sigma_{r} w^{\prime}(r)+\frac{2}{r} \sigma_{\theta} w\right) 4 \pi r^{2} d r+4 \pi r_{d}^{2} \sigma w\left(r_{d}\right)$.

The weak formulation for the hollow sphere is:

$0=\int_{\mathcal{B}_{d}}\left(\sigma_{r}^{\prime}+\frac{2}{r}\left(\sigma_{r}-\sigma_{\theta}\right)\right) w d V-\int_{\partial \mathcal{B}_{d}}\left(\sigma_{r}\left(r_{d}\right)-\sigma_{e}\right) w\left(r_{d}\right) d A+\int_{\partial \mathcal{C}_{d}}\left(\sigma_{r}\left(r_{c}\right)-\sigma_{i}\right) w\left(r_{c}\right) d A$,

that is,

$0=-\int_{r_{c}}^{r_{d}}\left(\sigma_{r} w^{\prime}(r)+\frac{2}{r} \sigma_{\theta} w\right) 4 \pi r^{2} d r+\sigma_{e} 4 \pi r_{d}^{2} w\left(r_{d}\right)-\sigma_{i} 4 \pi r_{c}^{2} w\left(r_{c}\right)$.

\section{References}

1. Dawson, C., Vincent, J.F.V., Rocca, A.M.: How pine cones open. Nature 390(6661), 668 (1997). https://doi.org/10.1038/37745

2. Burgert, P., Fratzl, I.: Actuation systems in plants as prototypes for bioinspired devices. Philos. Trans. R. Soc. A: Math. Phys. Eng. Sci. 367(1893), 1541 (2009). https://doi.org/10.1098/rsta.2009.0003 
3. Noblin, X., Rojas, N.O., Westbrook, J., Llorens, C., Argentina, M., Dumais, J.: The fern sporangium: a unique catapult. Science 335(6074), 1322 (2012). https://doi.org/10.1126/science.1215985

4. Erb, R.M., Sander, J.S., Grisch, R., Studart, A.R.: Self-shaping composites with programmable bioinspired microstructures. Nat. Commun. 4(10), 1712 (2013). https://doi.org/10.1038/ncomms2666

5. Llorens, C., Argentina, M., Rojas, N., Westbrook, J., Dumais, J., Noblin, X.: The fern cavitation catapult: mechanism and design principles. J. R. Soc. Interface 13(114), 20150930 (2016). https://doi.org/10.1098/rsif.2015.0930

6. Egunov, A.I., Korvink, J.G., Luchnikov, V.A.: Polydimethylsiloxane bilayer films with an embedded spontaneous curvature. Soft Matter 12, 45 (2016). https://doi.org/10.1039/C5SM01139F

7. Shi, Y., Zhang, J., Pan, L., Shi, Y., Yu, G.: Energy gels: a bio-inspired material platform for advanced energy applications. Nano Today 11(6), 738 (2016). https://doi.org/10.1016/j.nantod.2016.10.002

8. Peppas, N.A.: Hydrogels in Medicine and Pharmacy: Fundamentals, vol. 1. CRC Press, Boca Raton (2019). https://doi.org/10.1002/pi. 4980210223

9. Holmes, D.P., Roché, M., Sinha, T., Stone, H.A.: Bending and twisting of soft materials by non-homogenous swelling. Soft Matter 7, 5188 (2011). https://doi.org/10.1039/C0SM01492C

10. Kim, J., Hanna, J.A., Byun, M., Santangelo, C.D., Hayward, R.C.: Hayward, Designing responsive buckled surfaces by halftone gel lithography. Science 335(6073), 1201 (2012). https://doi.org/10.1126/science.1215309

11. Nardinocchi, P., Pezzulla, M., Teresi, L.: Steady and transient analysis of anisotropic swelling in fibered gels. J. Appl. Phys. 118(24), 244904 (2015). https://doi.org/10.1063/1.4938737

12. Pezzulla, M., Smith, G.P., Nardinocchi, P., Holmes, D.P.: Geometry and mechanics of thin growing bilayers. Soft Matter 12, 4435 (2016). https://doi.org/10.1039/C6SM00246C

13. Bertrand, T., Peixinho, J., Mukhopadhyay, S., MacMinn, C.W.: Dynamics of swelling and drying in a spherical gel. Phys. Rev. Appl. 6, 064010 (2016). https://doi.org/10.1103/PhysRevApplied.6.064010

14. Curatolo, M., Nardinocchi, P.: Swelling-induced bending and pumping in homogeneous thin sheets. J. Appl. Phys. 124(8), 085108 (2018). https://doi.org/10.1063/1.5043580

15. Hong, W., Zhao, X., Zhou, J., Suo, Z.: Theory of coupled diffusion and large deformation in polymeric gels. J. Mech. Phys. Solids 56(5), 1779 (2008). https://doi.org/10.1016/j.jmps.2007.11.010 http://www.sciencedirect.com/science/article/pii/S0022509607002244

16. Doi, M.: Gel dynamics. J. Phys. Soc. Jpn. 78(5), 052001 (2009). https://doi.org/10.1143/JPSJ.78.052001

17. Chester, S.A., Anand, L.: A coupled theory of fluid permeation and large deformations for elastomeric materials. J. Mech. Phys. Solids 58(11), 1879 (2010). https://doi.org/10.1016/j.jmps.2010.07.020

18. Lucantonio, A., Nardinocchi, P., Teresi, L.: Transient analysis of swelling-induced large deformations in polymer gels. J. Mech. Phys. Solids 61(1), 205 (2013). https://doi.org/10.1016/j.jmps.2012.07.010

19. Hong, W., Liu, Z., Suo, Z.: Inhomogeneous swelling of a gel in equilibrium with a solvent and mechanical load. Int. J. Solids Struct. 46(17), 3282 (2009). https://doi.org/10.1016/j.ijsolstr.2009.04.022

20. Cai, S., Lou, Y., Ganguly, P., Robisson, A., Suo, Z.: Force generated by a swelling elastomer subject to constraint. J. Appl. Phys. 107(10), 103535 (2010). https://doi.org/10.1063/1.3428461

21. Dai, H.H., Song, Z.: Some analytical formulas for the equilibrium states of a swollen hydrogel shell. Soft Matter 7, 8473 (2011). https://doi.org/ 10.1039/C1SM05425B

22. Illeperuma, W.R.K., Sun, J.Y., Suo, Z., Vlassak, J.J.: Force and stroke of a hydrogel actuator. Soft Matter 9, 8504 (2013). https://doi.org/10. 1039/C3SM51617B

23. Nardinocchi, P., Teresi, L.: Actuation performances of anisotropic gels. J. Appl. Phys. 120(21), 215107 (2016). https://doi.org/10.1063/1.4969046

24. Drozdov, A.D., Sommer-Larsen, P.: Swelling of thermo-responsive gels under hydrostatic pressure. Meccanica 51(6), 1419 (2016). https://doi.org/10.1007/s11012-015-0300-3

25. Zhang, J., Zhao, X., Suo, Z., Jiang, H.: A finite element method for transient analysis of concurrent large deformation and mass transport in gels. J. Appl. Phys. 105(9), 093522 (2009). https://doi.org/10.1063/1.3106628

26. Bouklas, N., Landis, C.M., Huang, R.: A nonlinear, transient finite element method for coupled solvent diffusion and large deformation of hydrogels. J. Mech. Phys. Solids 79, 21 (2015). https://doi.org/10.1016/j.jmps.2015.03.004

27. Curatolo, M., Nardinocchi, P., Puntel, E., Teresi, L.: Transient instabilities in the swelling dynamics of a hydrogel sphere. J. Appl. Phys. 122(14), 145109 (2017). https://doi.org/10.1063/1.5007229

28. Kang, J., Li, K., Tan, H., Wang, C., Cai, S.: Mechanics modelling of fern cavitation catapult. J. Appl. Phys. 122(22), 225105 (2017). https://doi.org/10.1063/1.5009747

29. Wang, H., Cai, S.: Cavitation in a swollen elastomer constrained by a non-swellable shell. J. Appl. Phys. 117(15), 154901 (2015). https://doi.org/10. $1063 / 1.4918278$

30. Wang, H., Cai, S.: Drying-induced cavitation in a constrained hydrogel. Soft Matter 11, 1058 (2015). https://doi.org/10.1039/C4SM02652G

31. Curatolo, M., Nardinocchi, P., Teresi, L.: Driving water cavitation in a hydrogel cavity. Soft Matter 14, 2310 (2018). https://doi.org/10.1039/ C8SM00100F

32. Curatolo, M.: Effective negative swelling of hydrogel-solid composites. Extrem. Mech. Lett. 25, 46 (2018). https://doi.org/10.1016/j.eml.2018. 10.010

33. Flory, P.J., Rehner, J.: Statistical mechanics of cross-linked polymer networks i. rubberlike elasticity. J. Chem. Phys. 11(11), 512 (1943). https://doi.org/10.1063/1.1723791

34. Flory, P.J., Rehner, J.: Statistical mechanics of cross-linked polymer networks ii. Swelling. J. Chem. Phys. 11(11), 521 (1943). https://doi.org/ $10.1063 / 1.1723792$

35. Gurtin, M.E., Fried, E., Anand, L.: The Mechanics and Thermodynamics of Continua. Cambridge University Press, Cambridge (2010) 
36. Tasker, A.L., Hitchcock, J.P., He, L., Baxter, E.A., Biggs, S., Cayre, O.J.: The effect of surfactant chain length on the morphology of poly(methyl methacrylate) microcapsules for fragrance oil encapsulation. J. Colloid Interface Sci. 484, 10 (2016). https://doi.org/10.1016/j.jcis.2016.08.058

37. Yang, X.L., Ju, X.J., Mu, X.T., Wang, W., Xie, R., Liu, Z., Chu, L.Y.: Core-shell chitosan microcapsules for programmed sequential drug release. ACS Appl. Mater. Interfaces 8(16), 10524 (2016). https://doi.org/10.1021/acsami.6b01277

38. Zhao, C., Zhang, G.: Review on microencapsulated phase change materials (mepcms): Fabrication, characterization and applications. Renew. Sustain. Energy Rev. 15(8), 3813 (2011). https://doi.org/10.1016/j.rser.2011.07.019

39. Kim, J., Kim, C., Song, Y., Jeong, S.G., Kim, T.S., Lee, C.S.: Reversible self-bending soft hydrogel microstructures with mechanically optimized designs. Chem. Eng. J. 321, 384 (2017). https://doi.org/10.1016/j.cej.2017.03.125

40. Huang, H., Petruska, A.J., Sakar, M.S., Skoura, M., Ullrich, F., Zhang, Q., Pan, S., Nelson, B.J.: Self-folding hydrogel bilayer for enhanced drug loading, encapsulation, and transport. In: 2016 38th Annual International Conference of the IEEE Engineering in Medicine and Biology Society (EMBC), pp. 2103-2106 (2016). https://doi.org/10.1109/EMBC.2016.7591143

41. Ideses, Y., Erukhimovitch, V., Brand, R., Jourdain, D., Salmeron, J., Hernandez, Gabinet, U., Safran, S., Kruse, S., Bernheim-Groswasser, A.: Spontaneous buckling of contractile poroelastic actomyosin sheets. Nat. Commun. 9, 2461 (2018). https://doi.org/10.1038/s41467-01804829-x

42. ter Schiphorst, J., Saez, J., Diamond, D., Benito-Lopez, F., Schenning, A.P.H.J.: Light-responsive polymers for microfluidic applications. Lab Chip 18, 699 (2018). https://doi.org/10.1039/C7LC01297G

Publisher's note Springer Nature remains neutral with regard to jurisdictional claims in published maps and institutional affiliations. 